\title{
A Molecular Interpretation of the Compaction Performance and Mechanical Properties of Caffeine Cocrystals : A Polymorphic Study
}

Aditya B. Singaraju ${ }^{1}$, Dherya Bahl' ${ }^{1}$, Chenguang Wang ${ }^{3}$, Dale C. Swenson ${ }^{2}$, Changquan Calvin Sun $^{3}$ and Lewis L. Stevens, ${ }^{1, *}$

1. Division of Pharmaceutics and Translational Therapeutics, College of Pharmacy, The University of lowa, lowa City, IA 52242

2. X-Ray Diffraction Facility, Department of Chemistry, The University of lowa, lowa City, IA 52242

3. Pharmaceutical Materials Science and Engineering Laboratory, Department of Pharmaceutics, College of Pharmacy, University of Minnesota, MN 55455. 


\section{Supporting Information}

Figure S1. Particle Size Distributions Obtained by Sieve Analysis for both forms of CAF:NBA.

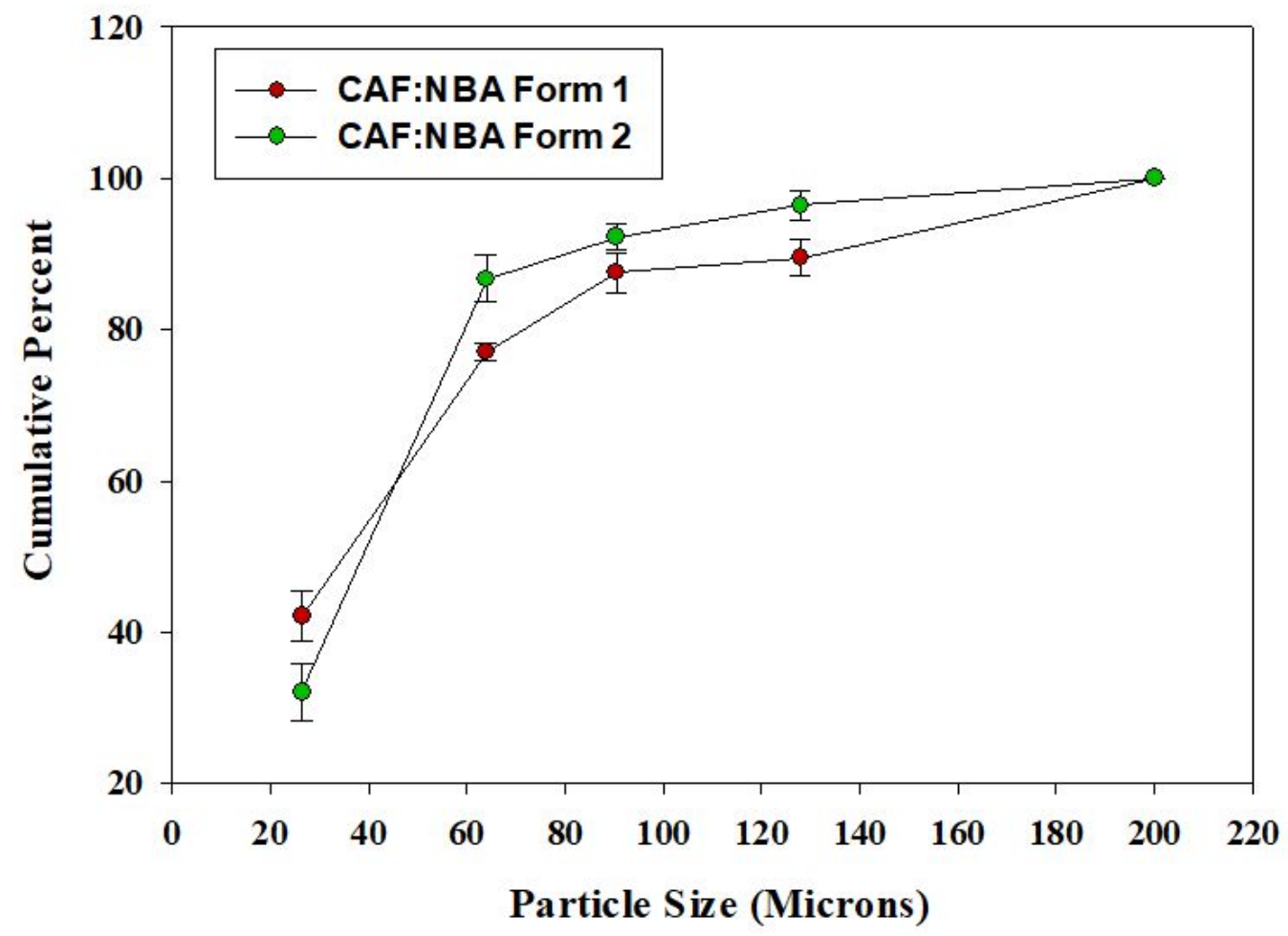

Figure S2. Out-of-die Heckel Plot for Polymorphic Forms of CAF:NBA.

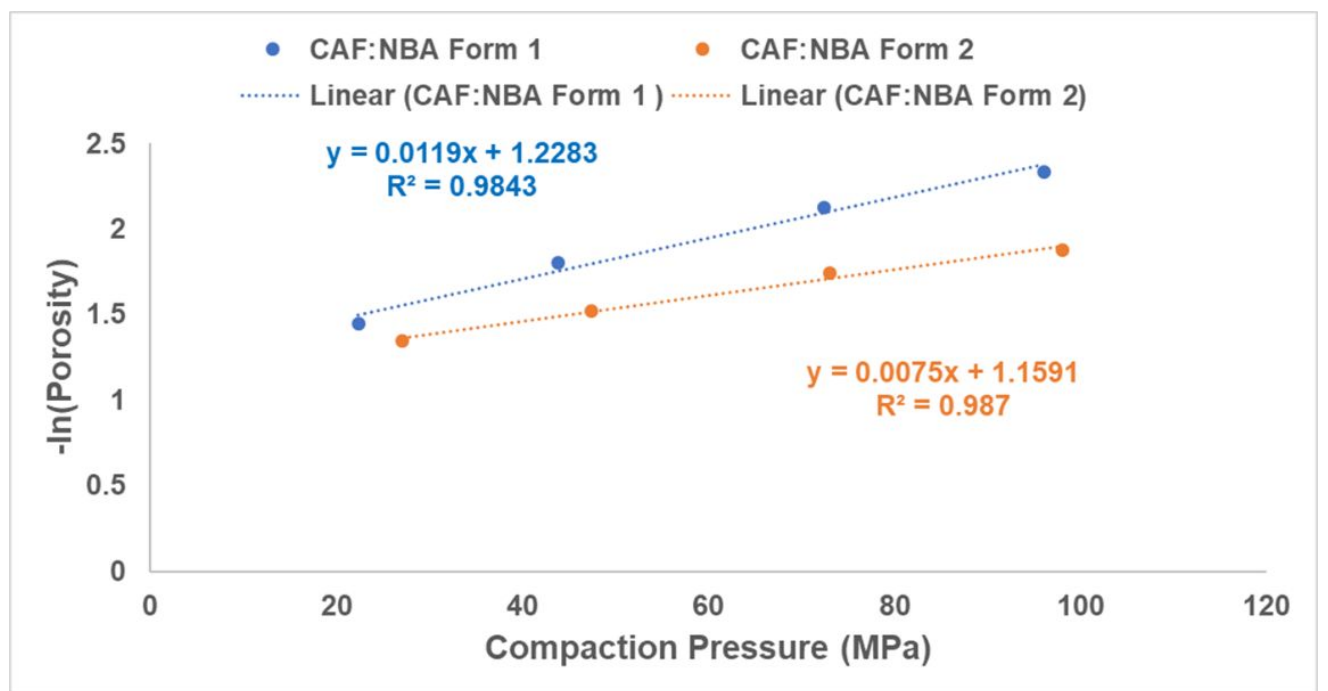


Figure S3. Ryshkewitch Analysis for Polymorphic Forms of CAF:NBA.

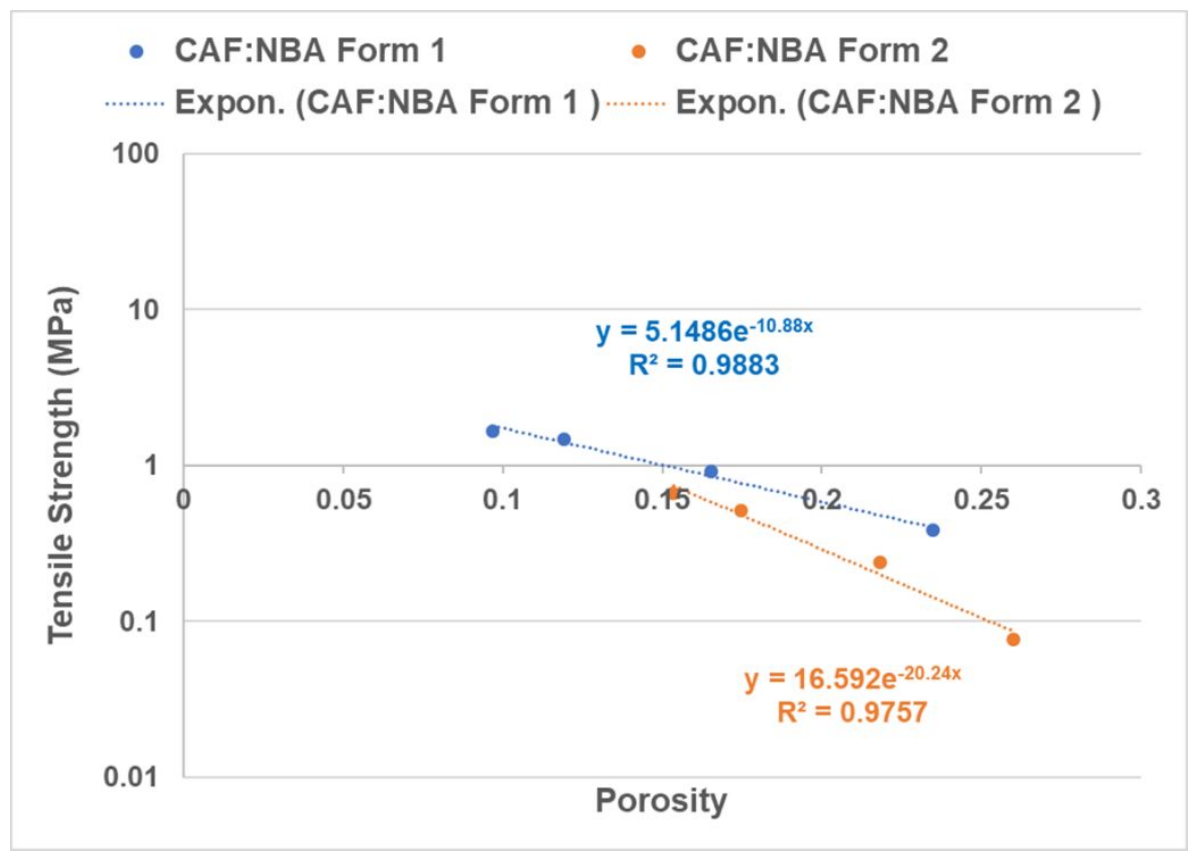

Figure S4. Overlay of PXRD patterns obtained from CAF:NBA polymorph slurry experiments.

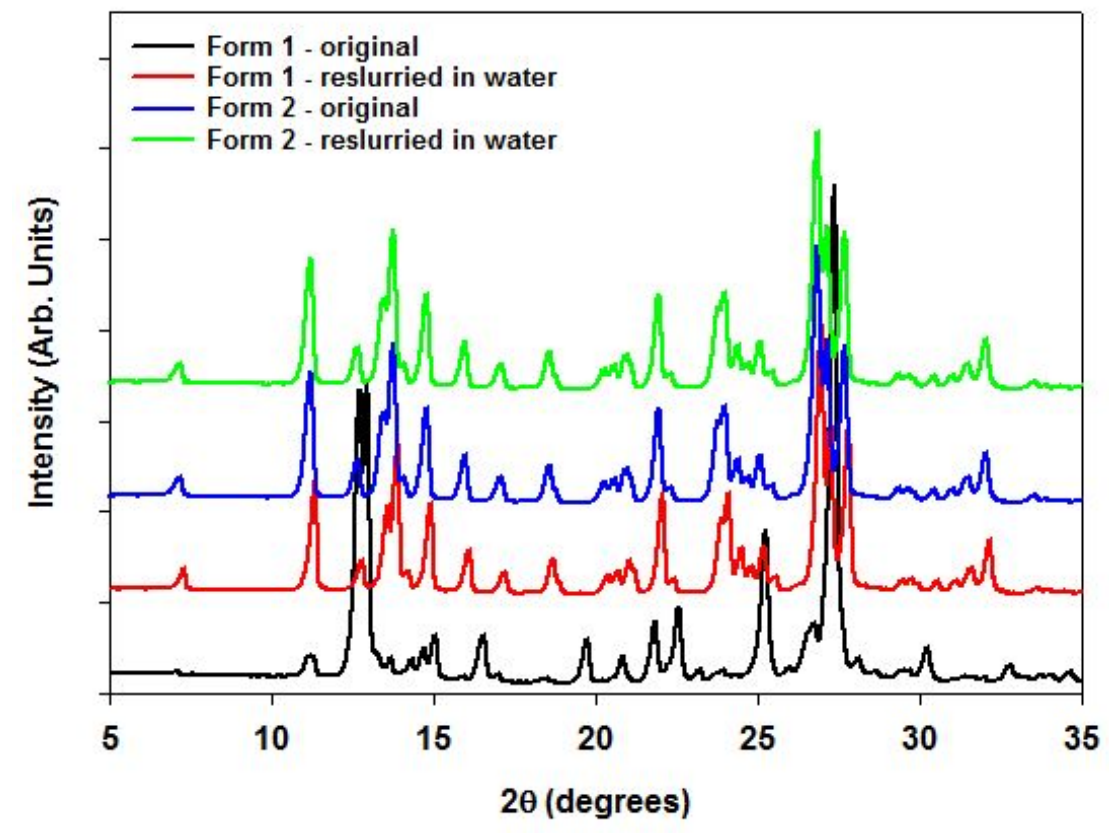


Table S1. Interaction energies for CAF:NBA Form 1 with symmetry operations

\begin{tabular}{|c|c|c|c|c|c|c|}
\hline Symop & $\mathrm{R}$ & $E_{\text {ele }}$ & $E_{p o l}$ & $E_{\text {dis }}$ & $E_{\text {rep }}$ & $E_{\text {tot }}$ \\
\hline - & 3.80 & 0.5 & 0.0 & -42.4 & 20.6 & -23.6 \\
\hline$x+1 / 2,-y+1 / 2, z$ & 8.90 & -4.0 & -1.0 & -2.2 & 0.2 & -6.7 \\
\hline - & 7.72 & -5.8 & -1.8 & -11.5 & 9.0 & -11.9 \\
\hline$-x+1 / 2, y+1 / 2,-z$ & 7.88 & -2.6 & -1.1 & -8.9 & 3.9 & -9.0 \\
\hline - & 7.85 & -11.2 & -0.5 & -10.4 & 8.1 & -16.3 \\
\hline - & 3.67 & -2.6 & -0.9 & -46.9 & 21.9 & -30.7 \\
\hline - & 8.21 & -7.3 & -2.6 & -9.5 & 10.6 & -11.3 \\
\hline - & 9.36 & -8.8 & -1.8 & -6.7 & 14.9 & -7.3 \\
\hline - & 7.94 & -2.3 & 0.0 & -8.4 & 10.5 & -3.3 \\
\hline$-x+1 / 2, y+1 / 2,-z$ & 8.71 & -0.0 & -1.1 & -1.7 & 0.0 & -2.3 \\
\hline - & 8.28 & -70.2 & -4.4 & -16.3 & 81.0 & -41.6 \\
\hline$-x,-y,-z$ & 9.43 & 0.5 & -0.2 & -4.3 & 1.6 & -2.5 \\
\hline - & 7.72 & -5.8 & -3.0 & -11.5 & 9.0 & -12.8 \\
\hline$-x,-y,-z$ & 9.05 & 2.0 & -1.3 & -2.7 & 0.0 & -1.2 \\
\hline$-x+1 / 2, y+1 / 2,-z$ & 9.92 & 0.6 & -1.0 & -1.8 & 0.2 & -1.6 \\
\hline - & 9.36 & -8.8 & -0.8 & -6.7 & 14.9 & -6.5 \\
\hline - & 8.28 & -70.2 & -0.6 & -16.3 & 81.0 & -38.8 \\
\hline$-x,-y,-z$ & 7.57 & -3.4 & 0.0 & -13.5 & 8.1 & -10.4 \\
\hline - & 3.80 & 0.5 & 0.0 & -42.4 & 20.6 & -23.6 \\
\hline
\end{tabular}




\begin{tabular}{|l|l|l|l|l|l|l|}
\hline$-x,-y,-z$ & 7.09 & -8.0 & -2.6 & -16.7 & 12.1 & -17.4 \\
\hline$x+1 / 2,-y+1 / 2, z$ & 8.95 & -8.8 & -1.1 & -9.6 & 5.3 & -15.2 \\
\hline- & 7.85 & -11.2 & -1.1 & -10.4 & 8.1 & -16.8 \\
\hline- & 3.67 & -2.6 & -1.4 & -46.9 & 21.9 & -31.1 \\
\hline- & 8.21 & -7.3 & -2.0 & -9.5 & 10.6 & -10.9 \\
\hline- & 7.94 & -2.3 & -1.5 & -8.4 & 10.5 & -4.4 \\
\hline
\end{tabular}


Table S2. Interaction energies for CAF:NBA Form 2 with symmetry operations

\begin{tabular}{|c|c|c|c|c|c|c|}
\hline Symop & $R$ & $E_{\text {ele }}$ & $E_{\mathrm{pol}}$ & $E_{\text {dis }}$ & $E_{\text {rep }}$ & $E_{\text {tot }}$ \\
\hline - & 5.61 & $\mid-6.6$ & 0.0 & $\mid-27.4$ & 19.6 & $\mid-18.7$ \\
\hline - & 8.29 & -4.0 & -1.7 & -8.2 & 3.8 & $\mid-10.3$ \\
\hline- & 7.62 & -21.4 & -4.7 & -11.9 & 19.2 & -24.5 \\
\hline- & 8.54 & -1.5 & -3.0 & -7.6 & 5.4 & -7.1 \\
\hline$-x,-y,-z$ & 5.55 & -2.5 & -2.9 & $\mid-33.4$ & 13.4 & -25.6 \\
\hline$x,-y+1 / 2, z+1 / 2$ & 9.45 & 0.2 & -1.4 & -4.8 & 2.8 & -3.4 \\
\hline$-x, y+1 / 2,-z+1 / 2$ & 7.44 & $\mid-1.4$ & -2.0 & -14.1 & 6.9 & $\mid-11.0$ \\
\hline- & 3.32 & -7.7 & -1.5 & -61.0 & 40.1 & -37.7 \\
\hline- & 8.29 & $\mid-89.7$ & -0.4 & -15.5 & 109.8 & -40.8 \\
\hline- & 8.05 & -4.4 & 0.0 & -4.3 & 3.8 & -6.0 \\
\hline- & 8.35 & $\mid-5.1$ & 0.0 & -6.4 & 3.9 & -8.5 \\
\hline$x,-y+1 / 2, z+1 / 2$ & 8.51 & -11.1 & -0.1 & -11.5 & 12.6 & $\mid-14.1$ \\
\hline$-x,-y,-z$ & 8.60 & 2.3 & -3.0 & -3.4 & 0.1 & -2.7 \\
\hline$-x,-y,-z$ & 5.00 & -3.8 & -0.8 & -23.3 & 10.7 & $\mid-18.3$ \\
\hline$x,-y+1 / 2, z+1 / 2$ & 8.38 & -7.9 & -0.0 & -4.3 & 3.8 & -9.7 \\
\hline- & 7.62 & -21.4 & -4.7 & -11.9 & 19.2 & -24.5 \\
\hline- & 8.54 & -1.5 & -1.8 & -7.6 & 5.4 & -6.3 \\
\hline$-x, y+1 / 2,-z+1 / 2$ & 8.03 & 0.7 & 0.0 & -3.3 & 0.7 & -1.7 \\
\hline$-x,-y,-z$ & 7.82 & -3.5 & -1.1 & -4.4 & 0.3 & -8.1 \\
\hline
\end{tabular}




\begin{tabular}{|l|l|l|l|l|l|l|}
\hline- & 5.61 & -6.6 & -2.5 & -27.4 & 19.6 & -20.6 \\
\hline$-x,-y,-z$ & 9.26 & 0.3 & -1.7 & -3.1 & 0.1 & -3.6 \\
\hline- & 8.29 & -89.7 & -2.8 & -15.5 & 109.8 & -42.6 \\
\hline- & 3.32 & -7.7 & -3.2 & -61.0 & 40.1 & -38.9 \\
\hline- & 8.29 & -4.0 & -1.8 & -8.2 & 3.8 & -10.3 \\
\hline- & 8.35 & -5.1 & -2.9 & -6.4 & 3.9 & -10.6 \\
\hline- & 8.05 & -4.4 & -1.4 & -4.3 & 3.8 & -7.1 \\
\hline
\end{tabular}

